\title{
DE L'AVENIR en général ET DE L'EAU en particulier
}

I

L Y A FORT À CRAINDRE que nos petits-enfants ne nous soient pas très reconnaissants... Sans être devin, un bref coup d'œil sur l'état du monde depuis 20 ans a en effet de quoi rendre modeste et pessimiste.

Modeste si l'on rapproche les belles idées humanistes de solidarité qui avaient alors cours, des réalisations auxquelles elles ont menées : déliquescence du tissu social dans l'ensemble des sociétés dites avancées, situation de plus en plus catastrophique à tous niveaux dans laquelle s'enfonce les pays auparavant sous-développés et depuis peu en développement (sic), destruction de plus en plus massive de la nature avec des conséquences encore incalculables (excès de $\mathrm{CO}_{2}$, trou d'ozone, désertification, pollution généralisée...).

Pessimiste lorsqu'on se rend compte que cela devrait vraisemblablement empirer, à l'image de la compétition technologico-économique qui fait rage entre les trois dernières grandes puissances, les États-Unis, le Japon et l'Europe (voir le «feuilleton » du Gatt !), et comme tend à l'envenimer la folie boursière qui se répand dorénavant partout dans le monde (100 fois plus de transactions boursières fictives que d'échanges marchands réels !).

Il faudrait être bien candide alors pour s'étonner de la multiplication exponentielle des accidents industriels majeurs et autres Tchernobyl, comme de la disparition de la mer d'Aral malheureusement sacrifiée sur l'autel d'un productivisme dépassé. Nos forêts, nos lacs, notre air même, sont bien peu de choses face à la construction d'empires technico-financiers transnationaux, reliés par les fils invisibles de la télématique toute-puissante, qui tentent de mettre le monde sous coupe réglée - sans se rendre compte que $\mathrm{du}$ fait de leurs agissements peu scrupuleux ledit monde risque d'être transformé en un désert brûlant ou bien noyé sous les eaux issues des glaces des pôles par la grâce d'un réchauffement généralisé provoqué pour l'essentiel par la fumée de nos voitures et de nos usines. 
Heureusement l'espoir survit toujours, et certains ont réussi à prendre suffisamment de recul pour s'apercevoir que la mondialisation de notre société occidentale conduisait la planète sur une pente plus que glissante. Ils ont ainsi émis l'idée en 1987, à travers le rapport rédigé par la commission Bruntdland (Our common future), qu'il serait peut-être possible d'harmoniser développement et environnement. Ce concept de "développement durable " suppose que la satisfaction des besoins des générations présentes ne doit pas compromettre celles des générations futures, autrement dit nos petits-enfants. C'est encore cette idée qui a été à l'honneur lors du Sommet de la Terre qui s'est tenu l'été 1992 à Rio de Janeiro. Dans cette révolution de notre façon de penser le monde - et en particulier les pays du Sud comme la nature en général -, non plus vacheà-lait mais bel et bien partenaire, l'eau tient une place centrale, elle qui conditionne toute vie et irrigue tous les compartiments de l'écosystème planétaire dont notre technocosme humain.

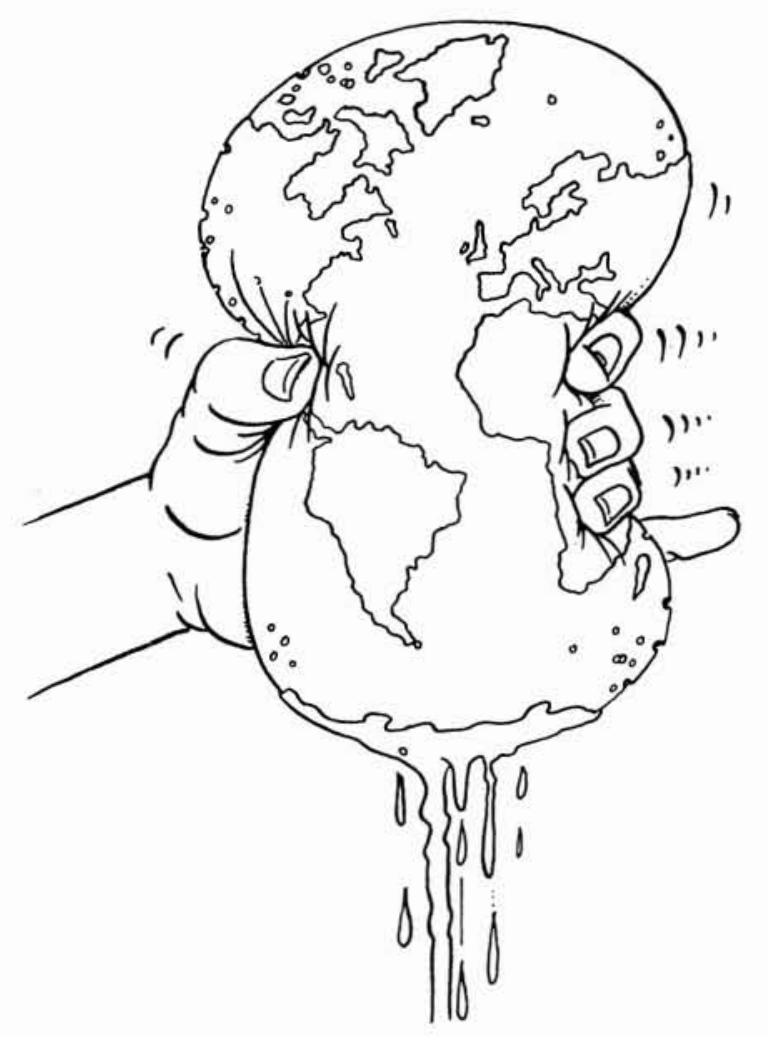

\section{L'eau,}

\section{entre écologie et politique}

\section{L'eau nous fait son cycle...}

Toute vie nourrit la vie. Le recyclage, tel est le principe de fonctionnement de la nature. Ainsi tout organisme une fois mort se décompose en éléments simples (carbone, hydrogène, azote...) qui serviront tôt ou tard à la construction d'un nouvel organisme. D'aucuns auraient dit « rien ne se crée, rien ne se perd, tout se transforme "! Et le moteur de cette immense machinerie, c'est l'eau, dont la circulation à l'intérieur des individus et entre les individus assure la répartition de ces éléments à tous les étages de l'écosystème.

L'eau est le seul élément qui peut cohabiter sous trois formes différentes en même temps, liquide (eau), solide (glace) et gazeuse 
(vapeur d'eau). Cette particularité en explique l'importance dans les réactions biochimiques (ainsi $63 \%$ du poids du corps humain est dû à l'eau) mais aussi sa présence dans les trois grands compartiments de la biosphère : atmosphère (vapeur), hydrosphère (eau) et lithosphère (glace, eau interstitielle).

Mais sur 1,6 milliard de $\mathrm{km}^{3}$ d'eau que l'on trouve sur la planète, les océans représentent l'écrasante majorité (1,38 milliard de $\mathrm{km}^{3}$ soit $97 \%$ ). Une conclusion immédiate à méditer : l'eau douce est une rareté ! C'est encore plus vrai si l'on s'intéresse à l'eau douce liquide qui ne représente que 0,275 million de $\mathrm{km}^{3}$ alors qu'il y a 16,5 millions de $\mathrm{km}^{3}$ d'eau congelée dans les glaciers et calottes polaires.

\begin{tabular}{|c|c|}
\hline Localisation ou forme & Volume en millions de $\mathrm{km}^{3}$ \\
\hline Océans & 1380 \\
Roches sédimentaires & 210 \\
Calottes glaciaires & 16,5 \\
Eaux souterraines & 0,25 \\
Eaux continentales de surface & 0,025 \\
Vapeur d'eau atmosphérique & 0,013 \\
\hline
\end{tabular}

\begin{tabular}{|c|c|}
\hline Nature de l'eau & Épaisseur * théorique » en m \\
\hline Océan mondial & 2700 \\
Calottes glaciaires & 100 \\
Eaux souterraines (nappes phréatiques) & 15 \\
Eaux continentales de surface & 0,4 \\
Vapeur d'eau atmosphérique & 0,03 \\
\hline
\end{tabular}

Source: F. Ramade (1984), Éléments d'écologie, p. 240.

En fait, l'origine de la circulation d'eau à travers la biosphère est l'évaporation de l'eau à la surface des océans sous l'action du soleil. Et pourtant, ce phénomène paraît a priori bien modeste puisque l'eau évaporée ne représente que $0,0009 \%$ du volume des océans, soit $13000 \mathrm{~km}^{3}$. Mais comme il se répète tous les jours puisque l'eau évaporée retombe sous forme de précipitations (dont près de $80 \%$ tombent directement «à la mer »!), c'est en fait $500000 \mathrm{~km}^{3}$ d'eau douce qui sont transportés annuellement par l'atmosphère.

Une vision anthropocentrique peut nous amener à concevoir le cycle de l'eau sous un autre angle : le «capital » serait constitué des $250000 \mathrm{~km}^{3}$ d'eau stockés dans les nappes souterraines et le « revenu » serait représenté par les précipitations annuelles terrestres $\left(110000 \mathrm{~km}^{3}\right) \ldots$ ou comment intégrer l'eau dans les calculs politiques. 


\section{L'eau en société}

Déjà les Romains réglementaient l'usage de l'eau en posant les fleuves comme publics et l'eau courante comme commune.

Mais l'eau en société est une eau polluée et c'est sur ce point que dès le $X X^{\mathrm{e}}$ siècle on va légiférer en France. La révolution industrielle va en effet véritablement inaugurer la pollution chronique et à grande échelle des eaux. C'est aussi à cette époque que Pasteur met le doigt sur la liaison entre eau, déchets et maladies. Ce qui entraîne les premières mesures relatives à l'amélioration de la qualité des eaux et notamment la séparation des réseaux privés et industriels. On crée aussi les systèmes d'égouts urbains débouchant dans les cours d'eau à l'aval des villes. Solution de prime abord séduisante, mais qui est malheureusement à l'origine de la pollution actuelle de nos rivières (phénomène "classique » en matière de lutte contre la pollution, dit du déplacement de pollution...).

La fin du XIX ${ }^{\mathrm{e}}$ siècle voit une véritable refonte du droit de l'eau en matière de régime des eaux, de salubrité publique et d'établissements classés, système législatif qui va perdurer durant toute la première moitié du $\mathrm{xx}^{\mathrm{e}}$ siècle.

\section{Évolution des normes sanitaires en France (d'après B. Baraque et T. Lavoux)}

Au $X I X^{e}$ siècle, les normes sanitaires ne concernaient que l'eau potable ; elles étaient très qualitatives et les qualités organoleptiques étaient confondues avec celles relatives à la santé publique. Depuis, les découvertes de Pasteur et de Koch sur les bactéries ont fait beaucoup évoluer les choses et, à l'heure actuelle, les normes en la matière s'appliquent à 62 paramètres en Europe et 80 aux ÉtatsUnis.

Dans le domaine de l'eau, on a trois grands types de normes : de procédé (ou de moyens), de résultats finaux (d'immission pour les anglo-saxons), et entre les deux des normes d'émission (visant un résultat intermédiaire). Au cours du temps, on est progressivement passé à des normes plus directement reliées aux objectifs à atteindre par la fixation de concentrations maximales.

Avec la loi de 1964, la France est allée très loin dans le processus de rationalisation en fixant les fameux "objectifs de qualité " concernant le milieu récepteur. Mais ceux-ci se sont révélés difficilement applicables immédiatement. On s'est donc progressivement réorienté vers des normes en matière de rejet indépendamment du pouvoir épurateur du milieu, dont la référence technique a été basée sur le procédé des " boues activées ". On est donc passé d'une norme d'immission à une norme de procédé, dont un des effets pervers a été le sur-engouement pour les boues activées aux dépens de toutes les autres techniques. II a alors été nécessaire de modifier de nouveau les normes de rejet pour favoriser le recours à un éventail plus large des techniques.

La Communauté européenne, dont les directives prennent le pas sur nos réglementations nationales (principe de subsidiarité), prépare une nouvelle norme sur la qualité écologique des milieux aquatiques, comprenant quatre classes et visant à faire remonter toutes les eaux d'une classe. Le choix semble se porter sur des bio-indicateurs même si l'ingénierie écologique n'est peut-être pas encore suffisamment mûre pour être vraiment fiable.

La vogue de l' "environnementalisme " pousse dans ce sens, mais est-il vraiment raisonnable de bâtir une politique de l'environnement sur des normes administratives? 
Puis, l'explosion économique et urbaine des années 1950 provoque naturellement un accroissement de la pollution qui donne lieu à des réglementations plus sévères (stations d'épuration) mais encore éparses et parfois irréalistes. Ce n'est qu'au début des années 1960 que l'on prend vraiment conscience de la nécessité de coordonner la législation, et c'est ainsi que la loi dite de 1964 va

\section{La pollution des nappes en France (d'après C. Guillemin)}

Alarmée par la dégradation croissante de la qualité des eaux souterraines, l'Académie des sciences a demandé en 1990 à son comité Environnement de faire le point sur la situation réelle. Le rapport d'étude en a été rendu public fin 1991 et a donné lieu à une table ronde en 1992.

\section{Les ressources souterraines françaises}

Elles représentent $60 \%$ des ressources nationales avec 200 aquifères d'importance régionale dont $25 \%$ sont captifs. Leur contenance est de 2000 milliards de $\mathrm{m}^{3}$ dont 100 sont en mouvement. 7 milliards de $\mathrm{m}^{3}$ y sont prélevés annuellement alors qu'on estime à 20 milliards de $\mathrm{m}^{3}$ les réserves utilisables chaque année. $61 \%$ de la population française est alimentée par ces eaux souterraines (et jusqu'à $95 \%$ dans certaines régions comme le Bassin parisien). Leurs principaux avantages sont la régularité de leur régime qui amortit considérablement les aléas climatiques et leur protection vis-à-vis des pollutions qui en fait des eaux de bonne qualité (même s'il existe quelques exceptions locales), dont la plupart ne nécessitent pas de traitement. Ce sont donc des réserves à la fois quantitatives et qualitatives pouvant jouer un véritable rôle stratégique.

\section{Les pollutions souterraines}

Les plus préoccupantes sont les pollutions diffuses dont les nitrates sont la cause principale, en croissance forte et régulière depuis 15 à 30 ans. 900000 personnes sont alimentées avec une eau contenant plus de $50 \mathrm{mg} / \mathrm{l}$ (norme sanitaire) de nitrates. 4 à 500 captages ont ainsi dû être fermés ces dernières années. En outre, on a constaté une concordance parfaite entre les zones à risque (culture industrielles, élevage) et celles à fortes teneurs en nitrates. Quelle que soit l'évolution des pratiques agricoles, on est d'ores et déjà certain que les concentrations continueront à augmenter dans les aquifères profonds non encore touchés par le front de nitrates.

D'autre part, on a constaté l'apparition de nitrates dans des nappes captives en provenance de nappes supérieures contaminées, du fait de forages d'irrigation mal réalisés. Enfin, 25 à $30 \%$ des forages contrôlés ont révélé la présence d'atrazine, pour laquelle la norme CEE est de $1 \mu \mathrm{g} / \mathrm{l}$.

Les nappes peuvent aussi être contaminées ponctuellement par exemple dans les grandes régions industrielles. Bien que localisées, ces pollutions sont le plus souvent très marquées comme en Alsace où 20 millions de tonnes de chlorure de sodium stockés à proximité de Mulhouse s'infiltrent dans la nappe à raison de 100000 t/an. La superficie polluée (avec des concentrations allant jusqu'à $300 \mu \mathrm{g} / \mathrm{l}$ près de Colmar) est évaluée à 20000 ha.

II existe aussi des pollutions métalliques (zinc, cuivre, plomb, mercure...) dont les plus fréquentes concernent le chrome hexavalent, nécessitant parfois la fermeture des captages publics. La fréquence des accidents industriels induit par ailleurs un grand nombre de pollutions par les hydrocarbures, dont la plus connue est celle de Petit-Couronne où, en 1990, $20000 \mathrm{~m}^{3}$ de supercarburant et de fuel sont partis dans la nappe.

Fréquents aussi sont les cas de pollution par les solvants organiques dont un des plus grave a été celui de Mulhouse en 1986 avec l'infiltration d'isomères du chloronitrobenzène sous d'anciennes décharges industrielles, qui a nécessité la fermeture d'un forage alimentant 35000 personnes.

En définitive, bien que le nombre de pollutions n'ait pas tendance à augmenter sensiblement, il reste que leur gravité, elle, s'accroît et que viennent au jour de nombreuses pollutions “ historiques " restées ignorées jusqu'à maintenant. Or, quelles que soient les mesures prises, certaines vont durer encore au moins un siècle! 
créer les agences financières de bassin qui, dans le cadre de leurs bassins hydrographiques, mettent en oeuvre les incitations financières permettant, par le jeu des redevances de pollution et de prélèvement, d'internaliser les coûts externes induits chez les autres usagers ou dans l'environnement. C'est l'application du principe pollueur-payeur, complété par des aides aux investissements propres. Les agences font de l'argent avec de l'eau sale et avec cet argent elle transforme l'eau sale en eau propre.

On a donc calqué la structure législative sur une unité de fonctionnement écologique : fait exceptionnel et malheureusement encore unique en matière de droit... Cette orientation a été renforcée en 1992 puisque les agences de bassin sont devenues les agences de l'eau, chargées aussi, des schémas d'aménagement à l'échelle de leur bassin et en relation avec les autres. On s'y préoccupe aussi bien de mise en valeur des eaux que de leur protection, qu'elles soient des cours d'eau, des lacs, des nappes souterraines ou des zones humides (étangs...). Un des aspects prioritaires dans un pays comme le nôtre, où, mis à part quelques crises, on ignore ce que c'est que de manquer d'eau, est bien évidemment la poursuite d'objectifs de qualité des eaux face aux multiples causes et risques de pollution.

\section{Du nord au sud, la qualité en question}

Une fois «en société », l'eau perçue dorénavant comme un service a bien des soucis face à nos appétits de consommation. Souillée par les ménages, enrichie plus que de raison en engrais et pesticides divers par les agriculteurs ou utilisée pour faire disparaître de façon discrète leurs déchets par les industriels, il n'est vraiment pas sûr qu'elle soit aussi propre en sortant que nous ne l'avons trouvée en entrant !

Et bien que la situation soit plus préoccupante dans nos sociétés justement dites «de consommation », tout n'est pas rose, loin s'en faut, dans les pays en développement confrontés à des problèmes sanitaires chroniques.

\section{Priorité à l'hygiène dans le tiers monde}

Constat alarmant à notre époque soi-disant moderne que celui dressé par l'Organisation mondiale de la santé : sur les 50 millions de morts annuelles, les trois quart sont dues à des maladies imputables à l'environnement et au mode de vie, et près de la moitié à des maladies infectieuses liées à l'eau de boisson.

C'est bien entendu le tiers monde qui est frappé. En Afrique notamment, que l'eau provienne des mares ou des puits tradition- 
nels aussi bien que des forages récents, elle se retrouve de toute façon polluée par des microorganismes (souvent d'origine fécale) au moment de sa boisson, soit que cette pollution ait été héritée de la source dans le premier cas $(100 \%$ des mares et $70 \%$ des puits traditionnels sont infectés), soit qu'elle soit apparue durant le transport et le stockage en jarres dans le deuxième cas. De plus, le souci légitime de la femme africaine - qui, il faut le souligner, effectue les travaux des champs en plus de tout le reste - de réduire au maximum sa fatigue lors du transport de l'eau lui fait logiquement préférer les sources les plus proches quelles qu'elles soient. Et si par miracle il s'agit d'un forage (on en compte en moyenne un pour 2000 personnes), ses multiples défaillances l'en détourne vite.

Malgré les ambitions affichées (mais si peu concrétisées) durant la Décennie internationale de l'eau, il est toujours urgent d'entreprendre un vaste programme d'éducation sanitaire des populations, associé à une campagne de modernisation et de multiplication des points d'eau (objectif : un forage pour 500 habitants), tout en généralisant la désinfection par l'eau de Javel, anti-microbien puissant et peu coûteux ( $8 \mathrm{~F}$ par an et par famille).

Quand

y'en a plus,

y'en a

encore...

\section{Industrialisés et pollués}

$\mathrm{Ne}$ soyons pas plus royalistes que le roi. Il est vrai que depuis les années 1960 et les premières vraies mesures à l'échelle de la pollution moderne (en particulier la création des agences de bassin), une «certaine » forme de cette pollution a diminué (les ma-

\begin{tabular}{|c|c|c|}
\hline TYPE DE POLLUTION & Nature ChimiQue & SOURCE \\
\hline $\begin{array}{l}\text { Physiques } \\
\text { Pollution thermique } \\
\text { Pollution radioactive }\end{array}$ & $\begin{array}{l}\text { Rejets d'eaux chaudes } \\
\text { Radio-isotopes }\end{array}$ & $\begin{array}{l}\text { Centrales électriques } \\
\text { Installations nucléaires }\end{array}$ \\
\hline $\begin{array}{l}\text { Chimiques } \\
\text { Pollution par les fertilisants } \\
\text { Pollution par des corps simples } \\
\text { toxiques } \\
\text { Pollution par les pesticides } \\
\text { Pollution par les détersifs } \\
\text { Pollution par les hydrocarbures } \\
\text { Pollution par les composés } \\
\text { organochlorés } \\
\text { Pollution par les autres composés } \\
\text { organiques de synthèse }\end{array}$ & $\begin{array}{l}\text { Nitrates, phosphates } \\
\text { Mercure, cadmium, plomb, } \\
\text { aluminium, arsenic... } \\
\text { Insecticides, herbicides, fongicides } \\
\text { Agents tensioactifs } \\
\text { Pétrole brut et ses dérivés } \\
\text { PcB, chlorophénols, solvants } \\
\text { chlorés } \\
\text { Très nombreuses molécules } \\
\text { (plus de } 7000 \text { ) }\end{array}$ & $\begin{array}{l}\text { Agriculture, (lessives) } \\
\text { Industrie, agriculture, pluies acides } \\
\text { Agriculture, (industrie) } \\
\text { Effluents domestiques } \\
\text { Industrie pétrolière, transports } \\
\text { Industries } \\
\text { Industries, divers usages, } \\
\text { dispersifs pour certaines }\end{array}$ \\
\hline $\begin{array}{l}\text { Biologiques } \\
\text { Matières organiques } \\
\text { fermentescibles }\end{array}$ & Glucides, lipides, protides & $\begin{array}{l}\text { Effluents domestiques, d'industries } \\
\text { agroalimentaires, du bois }\end{array}$ \\
\hline Microbiologiques & $\begin{array}{l}\text { Bactéries, virus entériques, } \\
\text { champignons }\end{array}$ & $\begin{array}{l}\text { Effluents urbains, élevages, } \\
\text { secteur agroalimentaire }\end{array}$ \\
\hline
\end{tabular}


tières en suspension et oxydables présentes dans les cours d'eau). Toutefois, il est aussi vrai que de nouvelles pollutions (plus graves ?) sont apparues ou se sont révélées, notamment avec la présence non seulement dans les eaux de surface mais aussi dans les eaux souterraines de métaux toxiques (très !) et de composés organiques issus de l'industrie chimique. Bien sûr, ces produits ne sont qu'en très faibles concentrations (traces), mais, manque de chance, ils possèdent une toxicité aiguë souvent associée à une grande durée dans le temps... ce qui leur permet en particulier de s'accumuler au long de la chaîne alimentaire. C'est ainsi qu'on trouve dans certains poissons du lac Michigan 2 à 4 millions de fois plus de РСB (un poison chimique parmi tant d'autres) que dans les eaux du lac qui reçoivent les effluents chargés de ce produit en provenance des usines chimiques.

«Pour vivre heureux vivons cachés ! » tel pourrait être le slogan du «pollueur français » qui a profité jusqu'aux années 1980 de la faible acuité apparente de ces «nouvelles pollutions» en France. Faiblesse à mettre au débit, non pas d'industriels plus scrupuleux qu'ailleurs en Europe, mais plutôt d'une industrie plus dispersée dans un pays relativement vaste et riche en cours d'eau. Mais la politique de l'autruche a ses limites et on s'est aperçu dès le milieu de la décennie 1980 que nos villes et nos industries rejetaient près de 9000 tonnes de matières oxydables dans les cours d'eau, les polluant totalement sur $5000 \mathrm{~km}$. Sans parler de la multiplication des décharges industrielles plus ou moins bien contrôlées dont un certain nombre contamine les nappes phréatiques sous-jacentes.

Ce n'est malheureusement souvent que sous la pression d'événements exceptionnels plus ou moins catastro-

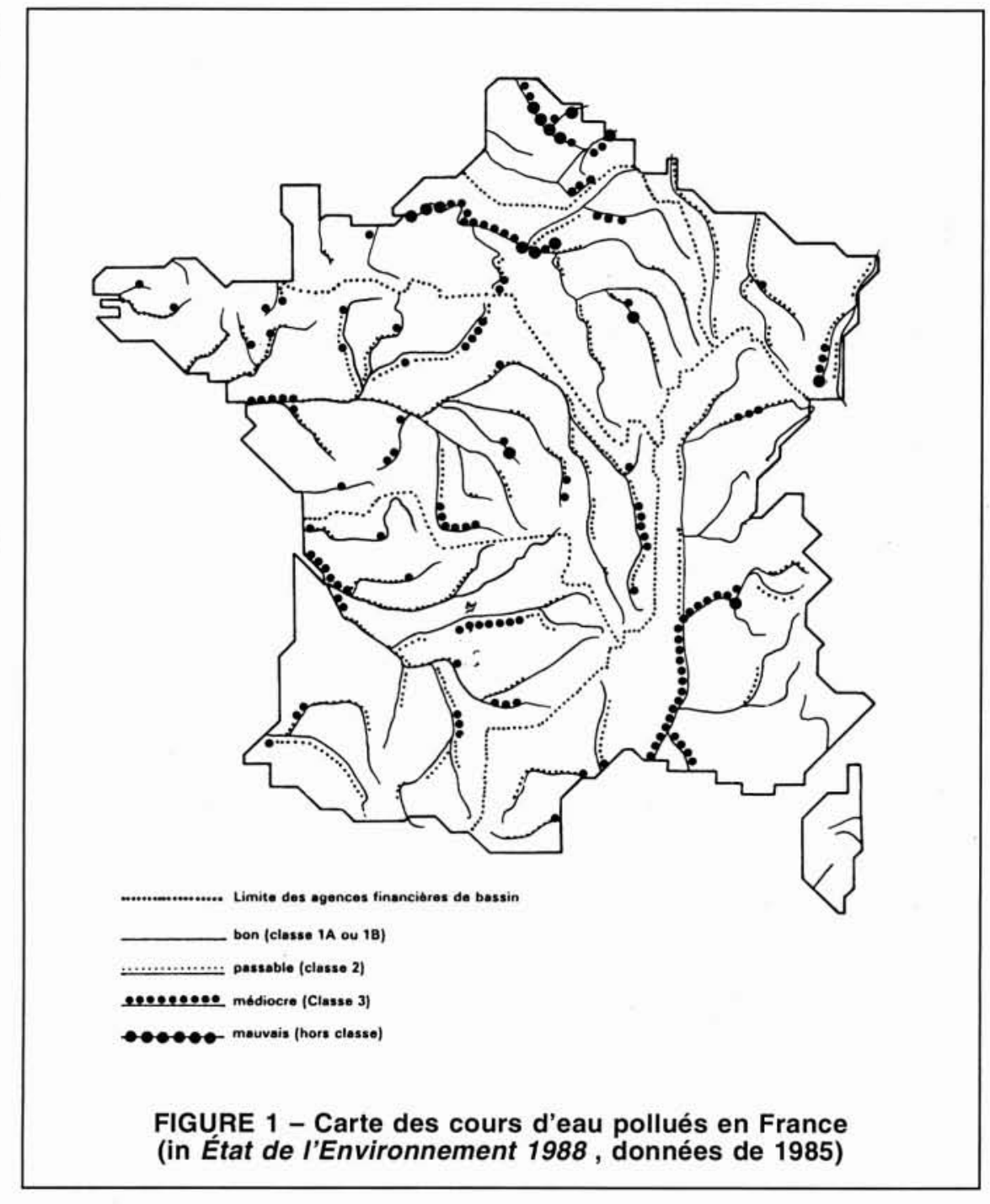


phiques que la société prend pleinement conscience des problèmes les plus graves. Ce type de démarche sociétale "à reculons " est tout à fait celle qui prévaut en matière d'environnement et d'eau en particulier. Sans s'attarder sur les multiples accidents industriels majeurs qui ont eu des conséquences dramatiques sur nos cours d'eau ou nos nappes ces dernières années, citons simplement l'incendie d'un entrepôt de la société Sandoz qui a provoqué en 1986 une pollution généralisée du Rhin de Bâle à la mer Baltique (y compris le réseau d'irrigation alsacien), la contamination du delta du Pô et des réseaux d'eau potable, toujours en 1986, par un désherbant chimique, l'atrazine, ainsi que l'accident Protex en 1988 qui a pollué la Loire et privé d'eau potable 200000 personnes à Tours pendant une semaine.

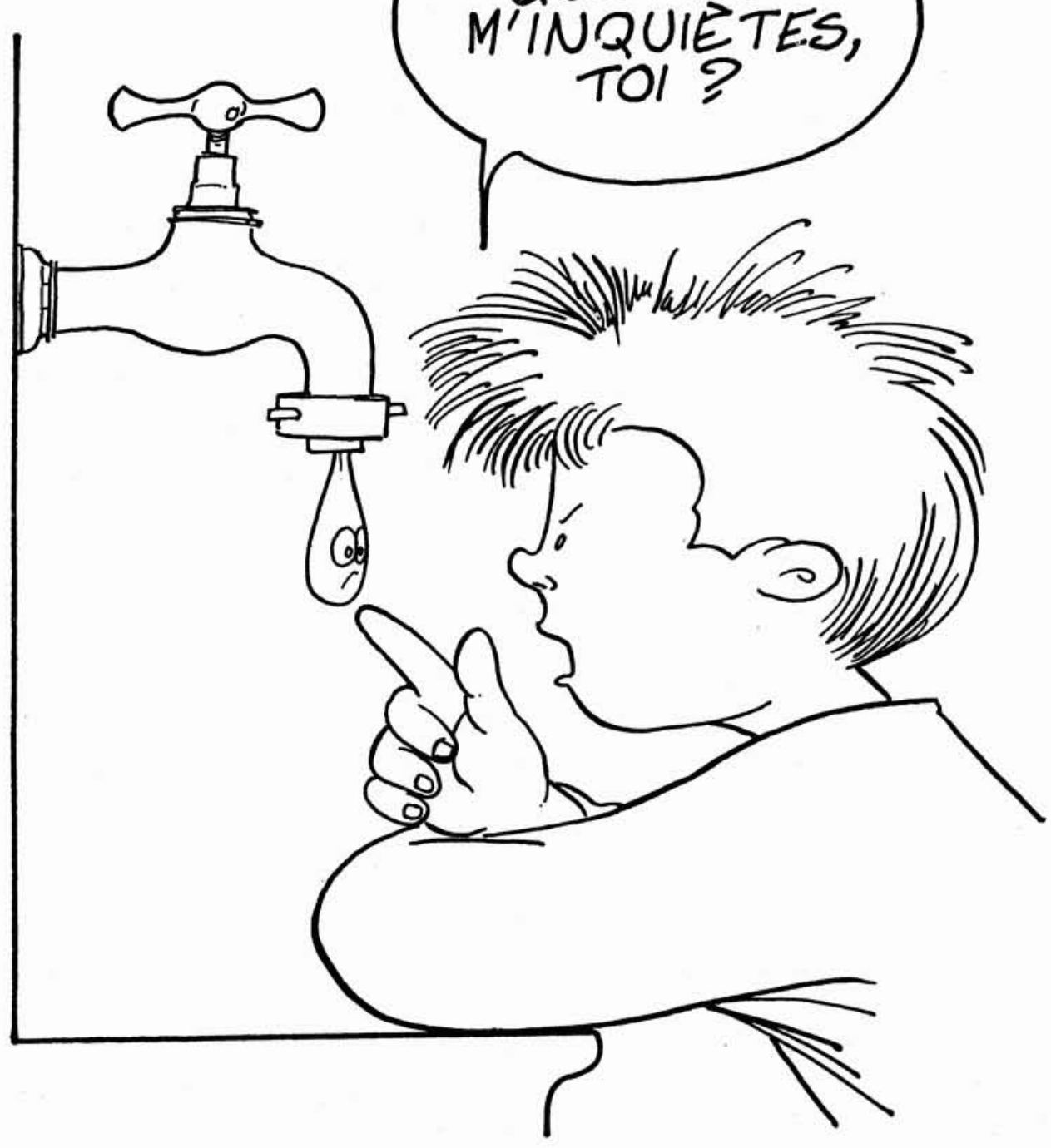




\section{Problèmes de quantité : nord contre sud ?}

La nature est-elle inégalitaire ? En matière d'eau, il est clair que la réponse est oui. Alors que certains pays sont régulièrement noyés sous les trombes d'eau, dans beaucoup d'autres on a parfois du mal à se souvenir de la dernière ondée déjà si loin dans les mémoires... Et pour couronner le tout, l'homme y met son grain de sel: que ce soit au Nord avec les pollutions industrielles ou au Sud avec la déforestation forcenée, il prend un malin plaisir à jouer à l'apprenti-sorcier en perturbant la marche «ancestrale » du cycle de l'eau.

\section{Géographie et géopolitique des disparités naturelles}

\section{Des différences de répartition...}

Bon an mal an, la machinerie climatique génère un flux de $40000 \mathrm{~km}^{3}$ d'eau (ce qui fait quand même 40000 milliards de $\mathrm{m}^{3}$ !) qui s'écoule sur l'ensemble des terres émergées du globe. Or, ne recevant que $6 \%$ des pluies mondiales, les zones arides et semi-arides ne bénéficient que de $2 \%$ de cet écoulement annuel. Première inégalité : la zonation des climats.

Deuxième point à considérer : l'étendue du pays dont dépend bien évidemment la taille de sa part du «gâteau hydrique » disponible dans une zone climatique donnée. Là, l'écart entre les diverses ressources «nationales " va de 1 à 50000 ! Les pays « les plus riches » possédant jusqu'à 5000 milliards de $\mathrm{m}^{3}$ d'eau par an. 9 pays seulement sont membres du club des "plus de mille fois milliardaires en $\mathrm{m}^{3}$ d'eau par an " (Brésil, Russie, Chine, Canada, Indonésie, États-Unis, Inde, Colombie et Zaïre), qui collecte à lui seul près des $2 / 3$ des ressources en eau mondiales ! A l'autre bout, le club des plus pauvres (quelques millions de $\mathrm{m}^{3}$ par an) compte bien sûr parmi ses membres les pays les plus petits du monde mais surtout les plus arides (Koweït, Libye...), dont un certain nombre d'îles (Malte). Par le jeu combiné de leur taille et de leur localisation, les pays industrialisés (en dehors des plus grands comme les États-Unis) sont au milieu du peloton (la France par exemple dispose de $165 \mathrm{~km}^{3}$ par an et le Japon de 547).

Mais il faut se préoccuper d'une troisième donnée : la population de chaque pays dont le nombre mis en rapport avec la ressource «nationale » brute conditionne la quantité d'eau dont peut disposer tout un chacun dans sa vie quotidienne. On s'aperçoit alors que la ressource en eau est partagée de manière encore plus inégale entre les hommes qu'entre les pays... Encore une fois, 10 pays forment un club où la quantité d'eau disponible par indi- 


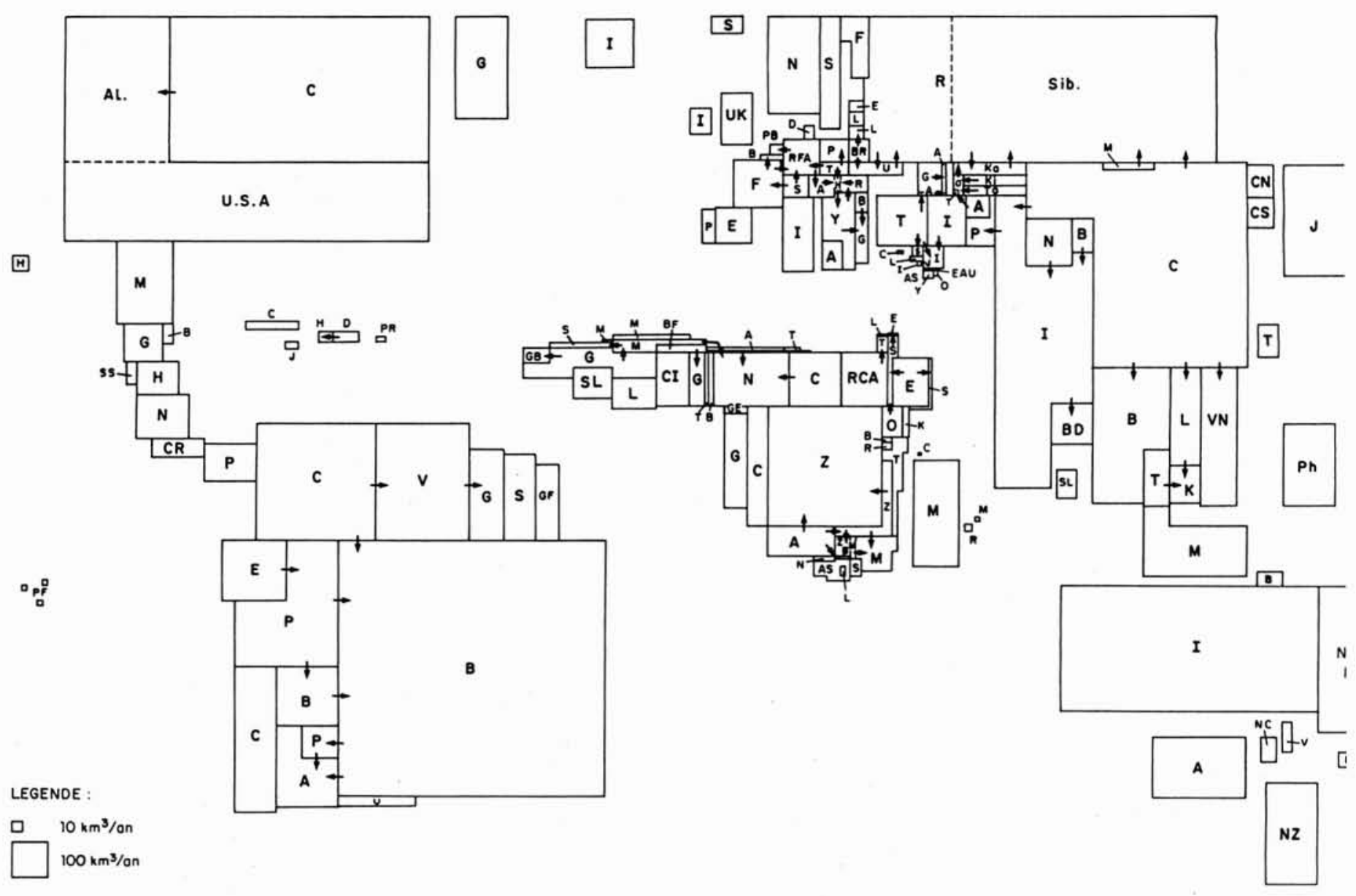

FIGURE 2 - Carte des ressources en eau internes de chaque pays (flux moyens annuels). Anamorphose cartographique (la surface de chaque pays est proportionnelle à ses ressources en eau)

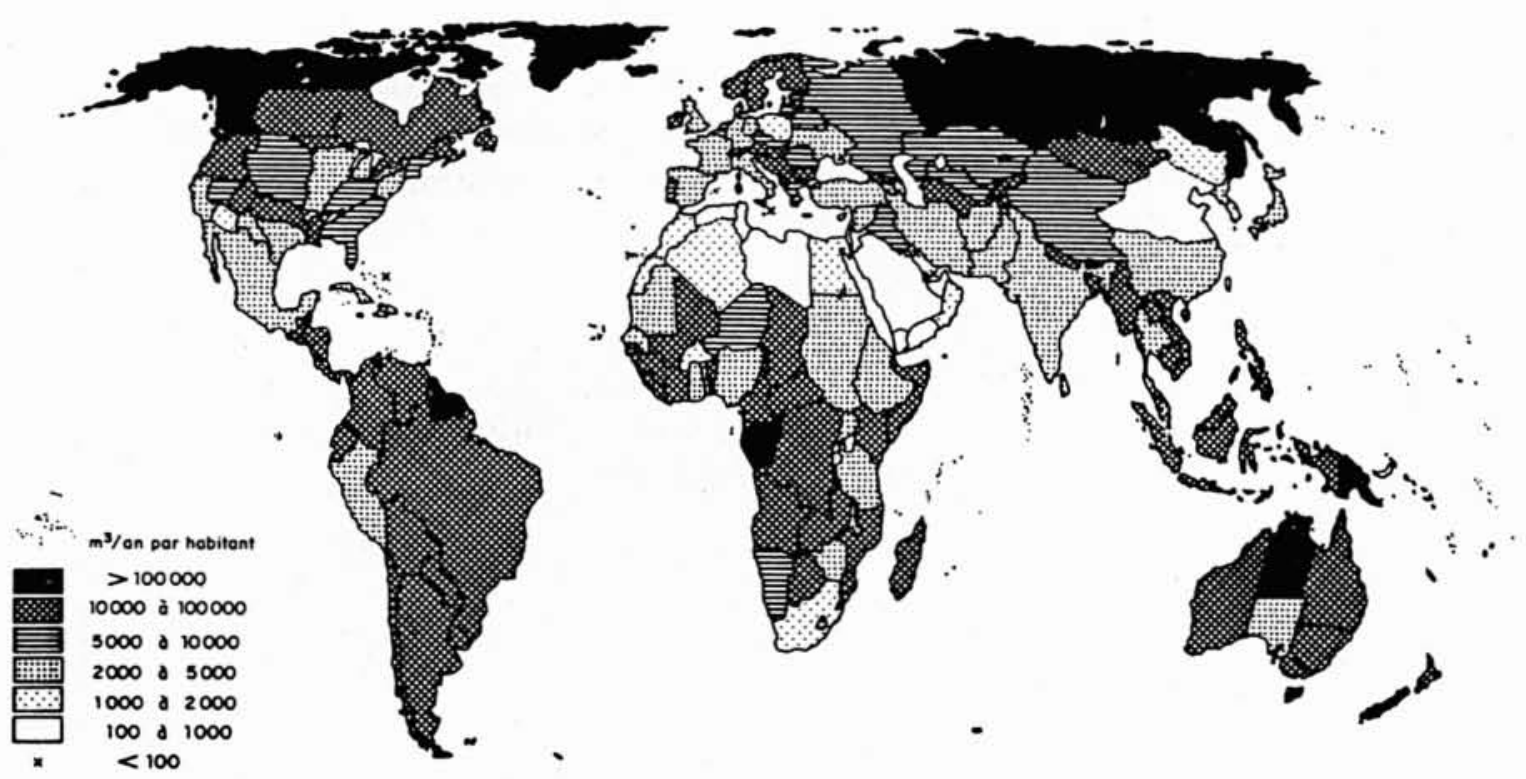

FIGURE 3 - Carte des ressources en eau naturelles, internes et externes (flux moyens annuels) par habitant (rapportées aux populations de 1985) 
vidu dépasse les $100000 \mathrm{~m}^{3}$ par an, alors que les plus faibles ressources par habitant se trouvent dans les pays arides ou insulaires (dont 8 pays arabes qui font partie des 12 plus pauvres). Selon cette optique, les pays tempérés développés sont toujours en situation intermédiaire, offrant à leurs habitants quelques milliers de $\mathrm{m}^{3}$ chaque année (3 360 exactement pour les Français).

\section{Ébauche d'une géographie hydroéconomique (d'après J. Margat)}

\section{Diversité des demandes}

Les concepts théoriques et normatifs de " besoins en eau ", utiles pour apprécier l'ampleur des restrictions ou des gaspillages éventuels, traduisent mal la variété des demandes effectives en eau suivant les usages, les pays ou les populations. Cette variété dépend en effet des caractéristiques climatiques (déficit hydrique estival, zone aride ou semi-aride...), de la taille des populations ainsi que de leur niveau de développement socio-économique (part de la demande industrielle, développement de l'irrigation...). Bien que ces demandes soient mal connues actuellement du fait de la faiblesse de l'arsenal statistique s'y rapportant, on peut grossièrement évaluer leurs différentes structures en fonction des principaux secteurs d'utilisation par grands espaces économiques. II en ressort notamment le poids largement prédominant de l'irrigation.

Rapportées aux populations, les demandes per capita sont très variables: de moins de $50 \mathrm{~m}^{3} / \mathrm{an}$ et par habitant à plus de $2000 \mathrm{~m}^{3}$ avec une moyenne mondiale de l'ordre de $600 \mathrm{~m}^{3} / \mathrm{an}$ et par habitant. Les demandes per capita les plus fortes se trouvent dans les pays où l'irrigation est primordiale (Irak, Pakistan, Madagascar, Iran, Égypte, Asie centrale ex-soviétique) ainsi que dans les pays industrialisés très développés (États-Unis, Canada), les deux facteurs pouvant se combiner (États-Unis, Espagne, Argentine, Australie). La part de l'irrigation peut être négligeable ou faible dans les pays industrialisés où la demande est modérée (RFA, Japon, Royaume Uni, Russie d'Europe) ou quand le poids de la population réduit le ratio (Chine, Inde). Enfin les demandes les plus faibles se rencontrent dans les pays à faibles ressources (zones arides: Libye, Tunisie ; îles de Malte et de Singapour) en dépit de la nécessité d'irriguer, mais aussi dans les pays peu développés de zone tropicale humide (Afrique centrale).
L'influence du niveau de développement sur les demandes en eau n'est pas aussi nette qu'on pourrait le penser, du fait du climat notamment. II y a ainsi peu de relations entre les quantités d'eau prélevées pour satisfaire les demandes des différentes branches d'activité et les contributions de celles-ci à la formation du PNB. Par contre, en comparant des pays de structure de demande similaire, la relation entre les prélèvements et le niveau de développement s'avère alors pertinente.

\section{Vers une géographie hydroéconomique}

Le croisement des gammes respectives de « richesse en eau " et de " dépenses en eau " rapportées aux populations définies précédemment permet de classer les pays suivant une typologie très différenciée. Le poids de la démographie dans la disponibilité des ressources par habitant va se traduire pour le $\mathrm{XXI}^{\mathrm{e}}$ siècle au mieux par une stabilisation mais plus généralement par une diminution de ces ressources qui sera plus marquée dans les pays où elles sont d'ores et déjà les plus faibles (pays arides). C'est aussi dans ces pays que l'accroissement des coûts de mobilisation sera le plus élevé du fait de l'appel à des ressources non-conventionnelles.

Conjointement à l'évaluation des niveaux de développement économique, ces facteurs devraient amener à une diminution de $50 \%$ des demandes per capita en Afrique septentrionale (Égypte, Libye) et de 15 à $40 \%$ au Maghreb, selon les prospectives du Plan Bleu à l'horizon 2025.

II devrait s'ensuivre une aggravation notable des tensions sur l'eau, avec une différence de plus en plus nette entre les pays à économie traditionnelle de l'eau, largement majoritaires, et ceux où l'eau devient de plus en plus un produit industriel. Mais partout les charges économiques des approvisionnements et de la conservation des ressources en eau devraient s'accroître sensiblement. 
Enfin dernier aspect de la question : l'origine de l'eau. Si l'eau de pluie appartient en principe à tout le monde, il n'en va pas de même de celle des fleuves, les plus grands notamment, dont l'origine est fréquemment dans un autre pays et qui changent parfois plusieurs fois de nationalités au long de leur cours. Ainsi, si seulement $8 \%$ de l' « eau française » est d'origine étrangère, l' « eau égyptienne » par exemple l'est à $99 \%$ ! Ce statut de «fournisseur » ou de "débiteur » en eau confère, on le conçoit, un enjeu géopolitique à ce fluide éminemment mobile et volatile...

\section{... potentiellement génératrice de conflits}

La « Guerre des robinets»

$\mathrm{Vu}$ la vitesse à laquelle crô̂t la population mondiale et donc ses besoins en eau, on peut d'ores et déjà imaginer la place que jouera la ressource « eau » dans la plupart des pays du Sud et particulièrement ceux qui sont plus ou moins arides. D'autant que certains d'entre eux exploitent déjà plus d'eau que la nature ne leur en fournit, et ce par le biais du recyclage des eaux usées, du dessalement de l'eau de mer ou du prélèvement dans des nappes souterraines fossiles.

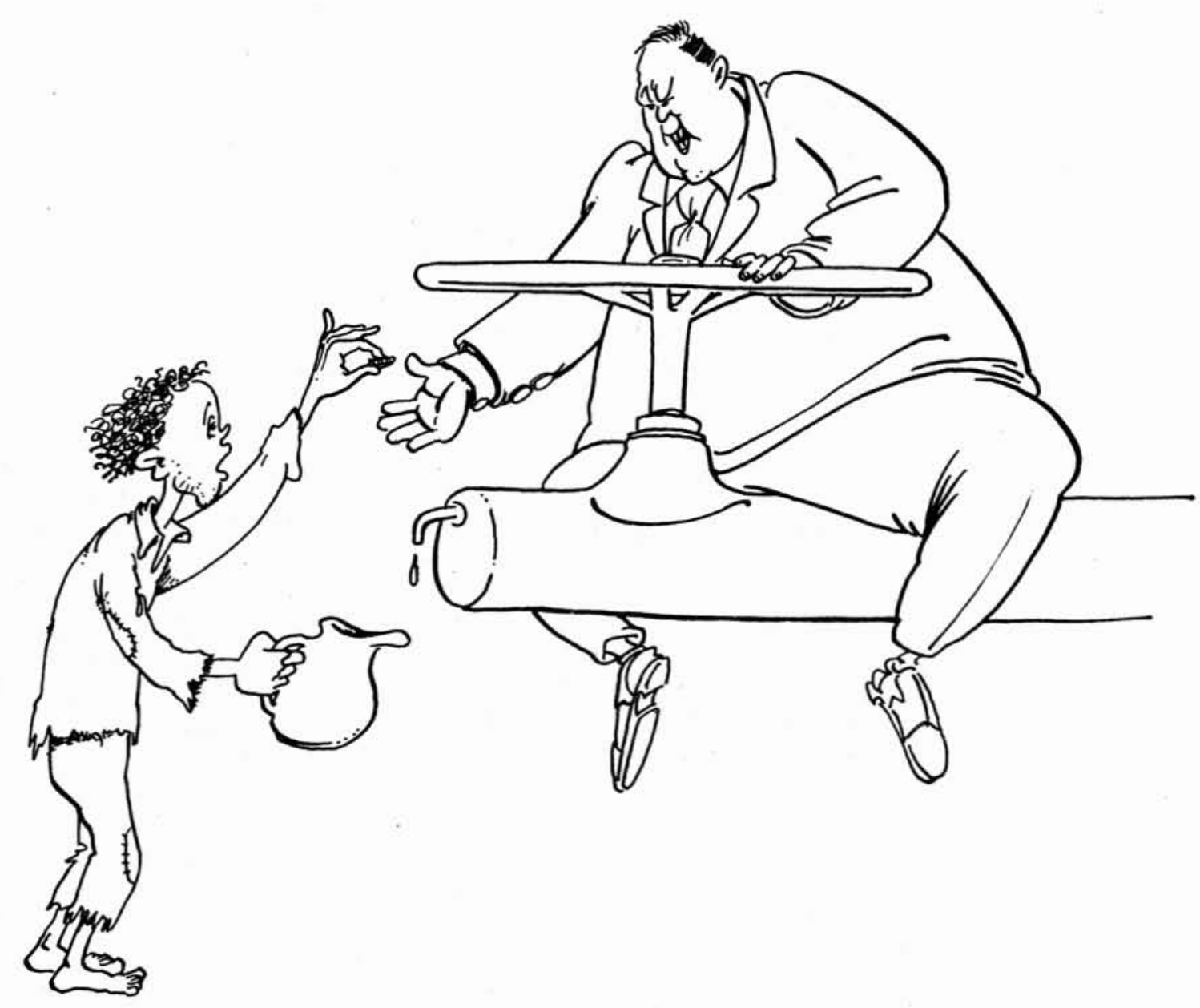


Ainsi, l'Arabie Saoudite par exemple utilise 4,5 fois sa ressource renouvelable ! Et dans tout le Moyen et le Proche-Orient on constate une tendance similaire à la surexploitation des - faibles - ressources en eau. Il s'ensuit logiquement des tensions permanentes et plutôt vives sur les trois grands - et quasi uniques fleuves de la région (le Jourdain, le Nil et le bassin Tigre-Euphrate), qui traversent tous plusieurs pays, non seulement très pauvres en eau mais aussi souvent en guerre les uns avec les autres !

La palme revient sans conteste au Jourdain qui irrigue une région vraiment très sèche mais surtout en conflit pratiquement permanent depuis 1948 puisqu'il s'agit de la Syrie, de la Jordanie, du Liban et d'Israël. A tel point qu'Anouar el-Sadate avouait au lendemain des accords de Camp David que désormais seule la question de l'eau était susceptible de relancer la guerre entre Israël et les pays arabes. Or à l'heure actuelle la question n'est toujours pas vraiment réglée.

C'est aussi le cas en ce qui concerne la gestion des eaux du Nil qui baigne 9 États et dont les principes ont été posés en 1959 lors de la construction du barrage d'Assouan. Bien qu'il soit prévu que les États situés à l'amont puissent revendiquer une part des eaux, cela n'a jamais été le cas depuis. Ce qui a fait le bonheur de l'Égypte, seul pays du bassin suffisamment stable politiquement pour avoir une gestion des eaux vraiment suivie. Mais la sécheresse qui sévit ces dernières années au Soudan et en Éthiopie risque à tout le moins de relancer le débat.

Enfin, cinq pays se partagent le bassin hydrographique du Tigre et de l'Euphrate. Depuis 1960, la Turquie a entrepris un projet "pharaonique " (17 centrales hydroélectriques, 21 barrages) visant à obtenir la maîtrise de ces deux fleuves pour irriguer 700000 ha. Elle a ainsi la possibilité de fermer le « robinet » Euphrate qui alimente la Syrie et l'Irak, ce qui inquiète légitimement ces deux pays. Des négociations sont toujours en cours afin d'obtenir que la Turquie assure un débit minimal de 500 à $700 \mathrm{~m}^{3} / \mathrm{s}$, ce qui est loin d'être acquis...

Ces quelques exemples soulignent la nécessité qu'il y aurait à adopter des principes équitables de partage des eaux, basés notamment sur la non dégradation des ressources à l'aval.

\section{Un cycle qui ne tourne plus rond}

L'homme a toujours eu besoin de domestiquer la nature pour la plier à ses exigences. En matière d'eau, il s'acharne à régulariser les flux, par exemple en construisant des barrages.

Pour spectaculaires qu'elles soient, ces constructions n'ont en fin de compte qu'un impact très local sur la circulation de l'eau. Même les plus grands barrages n'ennoyent au mieux que quelques centaines de kilomètres carrés et il est possible de minimiser certains de leurs impacts. C'est ce qui est fait en Guyane française, "Effet papillon», el Niño, dérive anthropogénique..., la météo poétise sur le barrage de Petit-Saut où l'on combat l'effet négatif de la 


\section{Rôle de l'océan et de EI Niño dans les variations interannuelles du régime des précipitations (d'après Y.-V. Serafini-Le Treut)}

L'eau possède une capacité calorifique et surtout une inertie thermique supérieure à la terre. On comprend donc aisément l'importance du rôle des océans qui recouvrent les $4 / 5^{\circ}$ de la planète et qui constituent de ce fait un gigantesque régulateur climatique dont l'impact est loin d'être négligeable même à l'intérieur des continents.

En dehors de l'énergie solaire reçue, dont les quantités varient à la fois dans le temps et dans l'espace, la température de surface de l'océan peut être modifiée par la présence de courants froids (ex. courant du Labrador) ou chauds (ex. El Niño).

El Niño - anomalie chaude de la température au milieu ou sur le bord Est du Pacifique équatorial -, dont l'importance a commencé à être soupçonné dès les années 1950, constitue de fait la plus forte oscillation naturelle du climat. Une éventuelle modélisation couplant océan et atmosphère permettrait vraisemblablement une bonne prédiction des épisodes $\mathrm{EI} \mathrm{Niño} \mathrm{et} \mathrm{donc}$ des précipitations en région tropicale, directement dépendantes d'un forçage thermique. En effet, un déplacement d'El Niño entraîne celui des grandes cellules climatiques dont les ascendances sont centrées sur les eaux chaudes et les forêts tropicales; ce qui provoque par contrecoup des sécheresses importantes dans les zones soumises aux descentes d'air sec, comme il y en eu en 1982-1983 en Indonésie, en Australie, dans le Nordeste brésilien, au Vénézuela et dans le Nord du Chili alors qu'à l'inverse le Nord de l'Argentine subissait de graves inondations...

Ce type de phénomène se retrouve sans doute sur l'océan Atlantique (variations pluviométriques en Afrique de l'Ouest) et sur l'océan Indien (mousson). II est certainement, bien que “ brouillé " par la grande variabilité naturelle du climat de ces zones, responsable d'une partie du comportement de l'atmosphère dans les régions tempérées. Toutefois, les possibilités de prédiction y semblent plus restreintes.

fermentation de la forêt tropicale noyée sur $310 \mathrm{~km}^{2}$, en construisant des dispositifs de réoxygénation de l'eau à l'aval.

Mais les véritables perturbations humaines du cycle de l'eau sont liées à l'effet de serre et à la déforestation.

Malheureusement, le fameux «effet papillon» (le battement d'aile d'un papillon en Australie serait susceptible de déclencher un cyclone de l'autre côté de la planète : c'est une image, bien sûr !) interdit, malgré l'arsenal méthodologique déployé (modélisation, théorie du chaos, informatique...), aux prévisions météorologiques de pouvoir évaluer avec une relative précision le temps qu'il fera au-delà d'un délai de quelques jours. Prévoir les conséquences de l'effet de serre, on en est encore loin

Une des solutions consiste à ne s'intéresser qu'aux variations saisonnières ou d'une année sur l'autre, ainsi qu'on le fait à propos de El Niño, le courant chaud atlantique qu'on soupçonne de plus en plus fortement de gouverner l'alternance sécheresses-inondations dans une grande partie du monde.

Afin de "rester dans le coup ", comme nombre de leurs confrères ayant maille à partir avec les phénomènes naturels, les météorologistes cherchent depuis quelques années à s'affranchir de ces contraintes par le recours à des modèles informatiques. Que ce soit pour prévoir l'évaluation de la couverture neigeuse des Alpes ou l'ampleur des modifications climatiques dont l'homme est responsable, ces modèles sont un peu comme les pulls tricotés 
main, ils ont des mailles trop larges ! Les données qu'ils exploitent ne sont pas assez fines pour pouvoir transcrire les phénomènes observés. Ils méconnaissent par ailleurs le rôle de l'océan, dont on commence seulement à concevoir la fonction de gigantesque régulateur climatique (par exemple en absorbant des quantités faramineuses de gaz carbonique).

\section{Des forêts qui partent en fumée...}

Autre élément naturel fondamental : la forêt, qui fixe l'eau et les sols, empêche le ruissellement et l'érosion... Sa disparition est le prélude à la désertification, particulièrement dans les zones tropicales où pourtant, dans les années 1980 déjà, 150000 km² étaient brûlés ou coupés chaque année.

Or, moins de forêts signifie moins de pluies, car dans ces régions les deux tiers environ des précipitations proviennent en fait de l'évaporation des arbres!

La pluie n'est pas seule à diminuer, l'eau qui s'infiltre dans le sol aussi car l'absence d'arbres favorise le ruissellement superficiel. Les nappes phréatiques reçoivent donc moins d'eau.

De surcroît, ce ruissellement accru provoque des crues et des inondations de plus en plus violentes et imprévisibles. C'est le cas par exemple au Bangladesh qui reçoit l'eau dévalant des flancs de plus en plus déboisés de l'Himalaya, et où les crues se répètent de plus en plus fréquemment chaque automne.

Autre conséquence du ruissellement, l'entraînement des sols. D'une part, il appauvrit les montagnes et l'agriculture y devient en conséquence plus difficile, et d'autre part il limite considérablement la durée de vie des barrages qui s'ensablent à grande vitesse, en tout cas beaucoup plus vite que leurs concepteurs ne l'avaient prévu. Et ce phénomène ne touche pas Source: F. Ramade, Les catastrophes écologiques que les pays du Sud. En France, le barrage de Serre-Ponçon, sur la Durance, s'envase deux fois plus vite qu'on ne le pensait.

Autre aspect du problème : la déforestation est pour l'essentiel menée par brûlage pour enrichir les sols ainsi défrichés pour l'agriculture. Dans les forêts gigantesques des régions tropicales, cela donne naissance à des incendies incontrôlables qui jour et nuit produisent d'énormes panaches de fumées, bien visibles par satellite et qui recrachent une quantité considérable de gaz carbonique dans l'atmosphère, contribuant ainsi notablement à l'effet de serre. 


\section{Modélisation des fluctuations climatiques \\ (d'après D. Duband)}

La constatation récente de l'augmentation du taux de $\mathrm{CO}_{2}$ atmosphérique de $20 \%$ sur les 100 dernières années amène les scientifiques à s'interroger sur d'éventuelles modifications du climat et surtout sur leurs échéances et leur quantification. Cela a conduit notamment à l'élaboration depuis 20 ans environ de modèles numériques chargés de simuler les processus physiques de l'atmosphère dans un premier temps, puis le fonctionnement du couple océanatmosphère, y compris les calottes glaciaires, les glaciers et les précipitations neigeuses.

Avec le temps, ces modèles s'affinent (intégration notamment de l'humidité et de l'évaporation sur les continents) mais ils ne donnent toujours accès qu'à des valeurs moyennes annuelles ou saisonnières rapportées à de grandes superficies. Les simulations s'améliorent en ce qui concerne les nuages mais butent sur la circulation océanique et ignorent l'influence de l'énergie solaire.

Quoi qu'il en soit, un des paramètres climatologiques les plus informatifs sur l'évolution du climat terrestre est la température de l'air qui semble varier corrélativement au taux de $\mathrm{CO}_{2}$ atmosphérique d'après l'analyse des carottes de glace prélevées en Antarctiques. Les autres paramètres accessibles sont, par ordre d'" explicativité " décroissante : les précipitations, les débits fluviaux, les niveaux des lacs et les bilans glaciaires. L'analyse de ces données est handicapée par la faible longueur des séries chronologiques s'y rapportant (au mieux 250 ans en Europe mais plus souvent 100 ans) et par l'existence d'un réseau de stations suffisamment dense depuis seulement 50 à 70 ans. Le recours à la dendroclimatologie et la corrélation avec des événements agricoles (ex. gels, déluges...) permet parfois d'établir des reconstitutions sur les 1000 dernières années.

\section{A propos de la température}

On a observé que depuis le $\mathrm{XII}{ }^{e}$ siècle on trouve en moyenne 9 hivers rigoureux par siècle. Et si certains scientifiques annoncent une augmentation de 0,3 à $0,6^{\circ} \mathrm{C}$ de la température de l'air au sol depuis 100 ans, d'autres, aux États-Unis notamment, le contestent à partir de 6000 séries remontant au $\mathrm{XIX}^{e}$ siècle.

II semble par ailleurs y avoir une certaine corrélation entre les redoux en Europe de l'Ouest et en Amérique du Nord et le cycle solaire (11 ans). Dans le même ordre d'idée, il est vraisem- blable que les cycles éruptifs jouent un rôle non négligeable (ex. éruption du Pinatubo).

Un des dangers de la modélisation est, suivant les données et les traitements, de faire apparaître des pseudo-cycles ou des pseudo-tendances. Ainsi il est possible que les constats apparents dégagés des analyses de séries chronologiques résultent en fait de processus markoviens relevant en fait du cheminement aléatoire. En définitive, on est à l'heure actuelle incapables de discerner dans l'éventuelle élévation de température de $0,4^{\circ} \mathrm{C}$, la part imputable à l'homme.

\section{A propos des précipitations}

Elles se caractérisent par un écart-type très important aussi bien au niveau spatial que temporel qui se traduit par l'impossibilité de dégager des tendances à long terme même en ayant recours à des analyses multidimensionnelles (ACP). Les alternances irrégulières d'années sèches et pluvieuses sont maintenant mises en grande partie sur le compte d'El Niño (voir encadré p. 390). Un effort important doit encore être consacré à l'enrichissement des banques de données.

\section{A propos des glaciers, fleuves, lacs et mers}

Les glaciers qui sont de bons " marqueurs " du climat semblent en Europe dans une phase de décrue. Mais il existe de nombreuses exceptions et leurs délais de réactions aux épisodes climatiques brouillent l'analyse.

II en va de même pour les fleuves dont les débits sont soutenus par les nappes phréatiques. L'étude du niveau des grands lacs mondiaux n'a pas fait ressortir non plus de tendances significatives.

Par contre, le niveau des mers (qui a varié aux cours des périodes de glaciation et de déglaciation de $+80 \mathrm{~m}$ à $-120 \mathrm{~m}$ ) semble monter régulièrement, au moins du fait du jeu des plaques tectoniques. Quant à l'influence d'un éventuel réchauffement (fonte des glaces et dilatation thermique), elle se traduirait environ par une montée des eaux de 1 à 1,5 $\mathrm{mm}$ par an. Globalement, aucun des paramètres climatiques pris en compte dans les modélisations ne peut permettre de dissiper les incertitudes quant à un réchauffement de notre planète lié à l'augmentation du taux de $\mathrm{CO}_{2}$ atmosphérique. 


\section{Vers une surchauffe climatique?}

Porté sur le devant de la scène par les controverses scientifiques à son sujet, l' "effet de serre " est devenu aussi familier à l'homme de la rue que le fameux «trou » d'ozone.

Sans rentrer dans les détails (voir encadré p. 405), il est toutefois bon de rappeler que ledit «effet de serre " est tout à fait naturel et qu'il est même à la base de la vie sur Terre puisque c'est ce phénomène (dû pour l'essentiel au gaz carbonique atmosphérique) qui est la cause de la relative clémence de la température sur notre planète.

Par contre, ce qui pourrait poser problème, c'est la «dérive anthropogénique » de l'effet de serre, à savoir la recrudescence de ce phénomène sous l'action de l'homme : industries au Nord, incendies au Sud. Il se pourrait en effet que les émissions artificielles massives de gaz carbonique mais aussi de méthane et autres gaz perturbent de façon notable les équilibres atmosphériques, modifiant des paramètres climatologiques comme les quantités ou la rythmicité des pluies ou encore la température de l'air. Autrement dit, tout le cycle de l'eau, fondement du climat terrestre tel que nous le connaissons, pourrait être bouleversé.

Si les conséquences en sont incalculables, l'ampleur de ces bouleversements l'est pour l'instant tout autant. Et même si des études prévoient une augmentation générale de température de $1,5^{\circ} \mathrm{C}$ à $5{ }^{\circ} \mathrm{C}$ en moyenne, les incertitudes concernant ces chiffres sont aussi grandes que les chiffres eux-mêmes. Ce qui déchaîne bien entendu les polémiques scientifico-politiques.

Toujours est-il que si cela s'avérait exact, les mers remonteraient de 0,25 à $1,5 \mathrm{~m}$, entre autres du fait de la fonte des glaces polaires, et que le volume des écoulements serait vraisemblablement modifié avec des conséquences plus ou moins prévisibles par exemple sur la production hydroélectrique.

\section{Conclusion}

En moins d'un siècle (1950-2050), l'humanité va avoir quadruplé, passant de 2,5 à 10 milliards d'individus selon les prévisions de l'ONu. Or, on a constaté que les besoins en eau augmente plus de deux fois plus vite que la taille de la population. Si l'on pense aussi aux menaces qui semblent peser sur notre climat, l'avenir paraît sombre en matière de satisfaction des besoins en eau. Surtout qu'à ces problèmes quantitatifs se surajoutent les problèmes qualitatifs, particulièrement dans le tiers monde.

La prise de conscience environnementale de ces dernières années sera-t-elle suffisamment profonde pour amener un véritable changement des mentalités ? La multiplication des conférences, des accords internationaux, des ouvrages, des dossiers et des ma- 
gazines abordant ces sujets peut à première vue inciter à être optimiste. L'eau semble d'ailleurs faire partie de ce "courant vert » qui traverse toute la société, industries comprises.

Mais la mise en place d'une réelle politique environnementale, qui bouscule un grand nombre de pratiques et de préjugés, implique des investissements colossaux, non directement rentables, surtout dans les pays du Sud. La vague verte sur laquelle surfent quelquefois nos dirigeants sera-t-elle suffisamment haute pour noyer toutes les réticences?

\section{Que ressort-il des débats?}

Manque d'eau ou manque d'argent?

Ça va mal sous nos nappes

\section{A changements globaux,} programmes globaux
- Premier sujet de préoccupation de l'assemblée, les inégalités de répartition mondiales présentées par J. Margat. C'est un des futurs problèmes majeurs pour une grande partie des pays du Sud, soit par insuffisance de ressource (Maghreb), soit par augmentation exponentielle de la population (Afrique). Or, les prospectives montrent qu'une baisse de la disponibilité de la ressource se traduira par une réduction de l'irrigation. Mais, même dans les pays pauvres en eau, on constate souvent des gaspillages importants, tant pour l'eau potable que pour l'irrigation ; des économies d'eau sont possibles, c'est une question de plomberie et d'organisation. Pourtant ces problèmes ne sont bien souvent qu'une question d'argent, ce qui repose la question de la solidarité après l'échec de la Décennie internationale de l'eau et, dans une moindre mesure, de la conférence de Rio.

- Deuxième point de débat, les menaces pesant sur la ressource " eau », évoquées par F. Ramade. On peut s'étonner à ce sujet du peu d'intérêt suscité par les techniques récentes d'économie de l'eau (par exemple irrigation au goutte-à-goutte). Sans doute sontelles difficile à mettre en œuvre dans les pays pauvres. Autre sujet d'inquiétude, l'état des nappes souterraines en France, qui s'avère beaucoup plus grave qu'on ne le soupçonne généralement. Mais le problème des solvants chlorés est encore trop récent pour en mesurer l'étendue réelle.

- En ce qui concerne les communications ayant trait aux changements climatiques, l'assistance s'est interrogée sur l'implication de la France dans le programme Mecca de simulation météo, à l'initiative des États-Unis. C'est en fait EDF qui y représente notre pays. La présentation des travaux portant sur les éventuelles conséquences (sur la neige et sur l'hydroélectricité) d'un réchauffement généralisé a provoqué des questions à propos des scénarios relatifs aux évolutions du taux de gaz carbonique atmosphérique (accroissement de température et politique de réduction des émissions). 
- Enfin, dernier sujet abordé, et non des moindres, les liaisons entre l'hydraulique rurale et la santé des populations qu'a exposées le $\mathrm{D}^{\mathrm{r}}$ Monjour. Point important, le rôle et les modalités de la chloration par l'eau de Javel. Cette problématique se retrouve aussi dans les grandes villes du tiers monde et nécessite des programmes spécifiques. Toute la démarche doit s'appuyer sur un effort éducatif particulier en direction des jeunes, les adultes semblant malheureusement d'ores et déjà " perdus ».

\section{Conclusions du président de séance Michel Mesny}

L'eau sera au $\mathrm{XXI}^{\mathrm{e}}$ siècle la ressource naturelle clé de notre planète. Comme on parle de géopolitique, on parlera d'hydropolitique. Le $\mathrm{Xx}^{\mathrm{e}}$ siècle a connu les guerres du pétrole, le $\mathrm{XXI}^{\mathrm{e}}$ siècle connaîtra les guerres de l'eau.

Face aux problèmes de l'eau, tant qualitatifs que quantitatifs, le monde apparaît profondément divisé.

D'un côté les pays nantis, ceux qui disposent, comme nous, de ressources en eau abondantes, plusieurs milliers de mètres cubes renouvelables par habitant et par an, ou ceux qui comme la Californie ont les capacités techniques et financières pour aller chercher une eau de plus en plus loin, de plus en plus chère. Ces pays irriguent leurs surplus agricoles. Ils peuvent se permettre le luxe de pratiquer une gestion patrimoniale de l'eau avec priorité à la protection de la qualité et des poissons. Les pénuries d'eau s'y limitent souvent à l'interdiction de laver les voitures ou d'arroser les pelouses. Dans ces pays, les normes d'eau potable atteignent une sévérité parfois délirante, à la limite du seuil de détection pour certaines substances chimiques soupçonnées de toxicité. Les réseaux d'eau potable sont alors sophistiqués, donc vulnérables et doivent être protégés pour éviter qu'ils ne constituent un point faible de ces pays.

De l'autre côté, au Sud, il y a les pays pauvres et secs, de plus en plus peuplés, où l'eau est une question de vie ou de mort, que la pénurie d'eau laisse sans espoir, sans avenir, avec leurs problèmes :

- problèmes alimentaires : pas assez d'eau pour irriguer, donc pas d'espoir d'autosuffisance alimentaire,

- problèmes sanitaires : actuellement c'est 20 millions d'hommes, 5 millions d'enfants, qui meurent chaque année parce qu'ils boivent de l'eau contaminée ; ce sont des centaines de millions de malades à cause de l'eau, un flot de diarrhées équivalent aux chutes du Zambèze !

Et malgré ces drames actuels, la population dans ces pays continue d'augmenter, avec comme conséquences : encore plus de déforestation, encore plus d'érosion, donc moins de sol et encore moins d'eau. C'est le cycle infernal. Rivières et puits s'assèchent, le désert avance. Pourtant la limitation des naissances reste en général un sujet tabou. Ces pays sont comme une voiture qui fonce 
à 100 kilomètres à l'heure vers un mur, le mur s'approche et le conducteur, au lieu de freiner, accélère.

Après la déception de la décennie internationale de l'eau, qui devait permettre à tout habitant de la planète de disposer de l'eau potable en 1990, une "goutte » d'espoir nous a été donnée par le docteur Monjour qui nous a parlé de l'activité de son association au Burkina-Fasso : formation des enfants, technique simple de désinfection de l'eau, avec une chloration de l'eau à la maison qui ne coûte que $8 \mathrm{~F}$ par an et par famille. Cette action sur le terrain porte ses fruits et a pu être entreprise grâce au syndicat des eaux d'Ile-de-France qui prélève $0,01 \mathrm{~F} / \mathrm{m}^{3}$ au profit de projets d'eau potable en Afrique, faisant ainsi participer tous les habitants d'Ilede-France à cet effort de solidarité... Exemple à suivre, où il apparaît que des initiatives à ras-de-terre, à condition qu'elles se multiplient, peuvent avoir plus d'impact pour la résolution des problèmes que de «grandes messes » planétaires. 